\title{
LOAD-BEARING CAPACITY OF PERFORATED TRAPEZOIDAL SHEETING
}

\author{
Thomas Misiek and Helmut Saal
}

\author{
Versuchsanstalt für Stahl, Holz und Steine, Karlsruhe Institute of Technology \\ e-mails: thomas.misiek@kit.edu, helmut.saal@kit.edu
}

Keywords: Trapezoidal sheeting, Corrugated sheeting, Perforation, Web crippling.

\begin{abstract}
Thin-walled perforated sheeting is used for sound absorption in buildings. The perforation may extend over the whole surface or only parts of the sheeting, e.g. webs. So far only reduction factors for the in-plane stiffness and the bending stiffness of fully perforated sheets with an equilateral triangular perforation pattern are included in EN 1993-1-3 and EN 1999-1-4. These factors only apply to perforation patterns in form of equilateral triangles, because of the isotropy assumed during derivation. Furthermore, the equations provide only information on stiffness and not on the stability behaviour of thin-walled components. The insufficient and misleading formulations of these regulations are overcome by the investigations presented here. Design rules for trapezoidal sheeting which are fully or partly perforated with equilateral triangular or square perforation patterns are given. The design rules take into account the beneficial effect of the post-buckling behaviour of the sheeting. They also cover web crippling at supports or under local loads.
\end{abstract}

Starting with the basic aspects of the load-bearing behaviour, the parameters required for capturing the influence of the perforation are defined and determined for the different perforation patterns. By introducing these parameters into the design procedures for the calculation of the load-bearing capacity of thin-walled structural components being at risk of buckling failure, the use of these procedures is expanded to fully perforated structural components. Numerical investigations with the finite-elementsmethod allow the verification of these theoretically derived calculation procedures.

Based on comprehensive numerical and experimental investigations on web crippling, it can be shown for fully and partially perforated trapezoidal profiles, that the influence of the perforation can be captured by multiplying the capacities according to EN 1993-1-3 and EN 1999-1-4 with a factor $C_{p}$ for fully perforated profiles and $C_{t p}$ for partially perforated profiles. A calculation procedure for these factors is given.

The recalculations of more than 600 experimental tests with perforated trapezoidal and corrugated sheeting which were performed since the 1980s at the University of Karlsruhe show the good correlation between the calculation procedure and the test results.

\section{INTRODUCTION}

The rules and standards recently given in EN 1993-1-3 [1] and EN 1999-1-4 [2] include approaches for determining reduction factors for membrane or bending stiffness as a basis for a verification by calculation of the cross-section. Due to lack of other published investigations, the basis of these equations are numerical investigations by Schardt and Bollinger [3]. These equations, however, only apply to perforation patterns in form of equilateral triangles, because of the isotropy assumed during derivation. 
Furthermore, the equations provide only information on stiffness and not on the stability behaviour of thin-walled components. This was the reason for starting the investigations presented here. Corresponding to the envisaged field of application of the results, the investigations restrict to perforation patterns with a continuous arrangement as equilateral triangle or as a square (Figure 1), with equal hole diameters $d$ and spacings $c$. The perforation pattern is defined by the hole diameter $d$ and the distance of the hole centres $\mathrm{c}$. With regard to their mechanical properties, triangular perforation patterns are isotropic - the alignment of the perforation patterns to the load direction does not play any role. Based on the orthotropy, orientation must be considered for the quadratic perforation pattern. For this pattern, the most prevalent orientation is the alignment of the row holes parallel and rectangular or with an inclination of $45^{\circ}$ to the main stress directions.

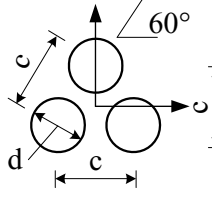

(a)

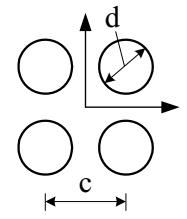

(b)

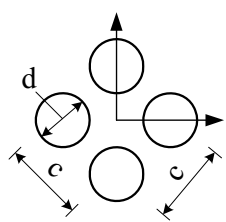

(c)

Figure 1: Perforation patterns

\section{STIFFNESS}

The perforation reduces the stiffness of the sheet. For the orthotropic sheet with the quadratic hole pattern, the stiffness matrix is:

$$
\left[\begin{array}{l}
m_{11} \\
m_{22} \\
m_{12}
\end{array}\right]=\left[\begin{array}{ccc}
K_{11, p} & K_{12, p} & 0 \\
K_{12, p} & K_{11, p} & 0 \\
0 & 0 & K_{44, p}
\end{array}\right] \cdot\left[\begin{array}{c}
-\kappa_{11} \\
-\kappa_{22} \\
-2 \cdot \kappa_{12}
\end{array}\right]
$$

In the present case with equal hole diameters $\mathrm{d}$ and spacings $\mathrm{c}$, the following applies in addition:

$$
K_{11, p}=K_{22, p}
$$

The reduced stiffness is expressed with the normalised effective bending stiffnesses $\mathrm{k}_{11}, \mathrm{k}_{12}$ and $\mathrm{k}_{44}$ related to the bending stiffness of the unperforated sheet.

$$
\begin{aligned}
& K_{11, p}=\frac{E_{1}}{1-v_{12} \cdot v_{21}} \cdot \frac{t^{3}}{12}=k_{11} \cdot K_{11}=k_{11} \cdot \frac{E}{1-v^{2}} \cdot \frac{t^{3}}{12} \\
& K_{12, p}=\frac{v_{12} \cdot E_{2}}{1-v_{12} \cdot v_{21}} \cdot \frac{t^{3}}{12}=k_{12} \cdot K_{12}=k_{12} \cdot \frac{v \cdot E}{1-v^{2}} \cdot \frac{t^{3}}{12}
\end{aligned}
$$

and

$$
K_{44, p}=G_{12} \cdot \frac{t^{3}}{12}=k_{44} \cdot K_{44}=k_{44} \cdot G \cdot \frac{t^{3}}{12}=k_{44} \cdot \frac{E}{2 \cdot(1+v)} \cdot \frac{t^{3}}{12}
$$

As in [3], it is assumed that the membrane and bending stresses are decoupled and the effective stiffnesses are determined with the basic elements of which the perforated sheet is composed. In addition to [3], on the one hand also quadratic perforation patterns are considered and on the other hand, to get a complete stiffness matrix, also the stiffnesses $\mathrm{K}_{12, \mathrm{p}}$ and $\mathrm{K}_{44, \mathrm{p}}$ are determined.

The related effective bending stiffnesses decrease with increasing ratio $\mathrm{d} / \mathrm{c}$. Figure 2 shows in addition, that the plate thickness $t$ (expressed by the ratio $t / c$ ) also has an influence on the bending stiffness. Figure 2 shows also, that for a constant $\mathrm{d} / \mathrm{c} \mathrm{k}_{\mathrm{ij}}=\mathrm{K}_{\mathrm{ij}, \mathrm{p}} / \mathrm{K}_{\mathrm{ij}}$ may be approximated by the geometry 
function $\Gamma$. This function $\Gamma$ describes the ratio of torsion stiffness to bending stiffness. Thus, only effective bending stiffnesses for a very small and a very large ratio $t / c$ or $t / c_{0}$ have to be determined between which an interpolation is performed by means of the geometry function

$$
\Gamma\left(\frac{t}{c}\right)=\left\{\begin{array} { c } 
{ 3 \cdot ( \frac { 1 } { 3 } - \frac { t } { 5 \cdot c _ { 0 } } ) } \\
{ 3 \cdot ( \frac { 1 } { 3 } - \frac { c _ { 0 } } { 5 \cdot t } ) \cdot ( \frac { c _ { 0 } } { t } ) ^ { 2 } }
\end{array} \text { for } \left\{\begin{array}{l}
\frac{t}{c_{0}} \leq 1 \\
\frac{t}{c_{0}}>1
\end{array}\right.\right.
$$

The related bending stiffnesses between which an interpolation has to be performed are in the following called $\mathrm{k}_{\mathrm{ij} \text {,mint }}$ and $\mathrm{k}_{\mathrm{ij} \text {,maxt }}$. Then, the following applies:

$$
k_{i j}\left(\frac{d}{c}, \frac{t}{c}\right)=\left[k_{i j}\left(\frac{d}{c}\right)\right]_{\max t}+\left\{\left[k_{i j}\left(\frac{d}{c}\right)\right]_{\min t}-\left[k_{i j}\left(\frac{d}{c}\right)\right]_{\max t}\right\} \cdot \Gamma\left(\frac{t}{c}\right)
$$

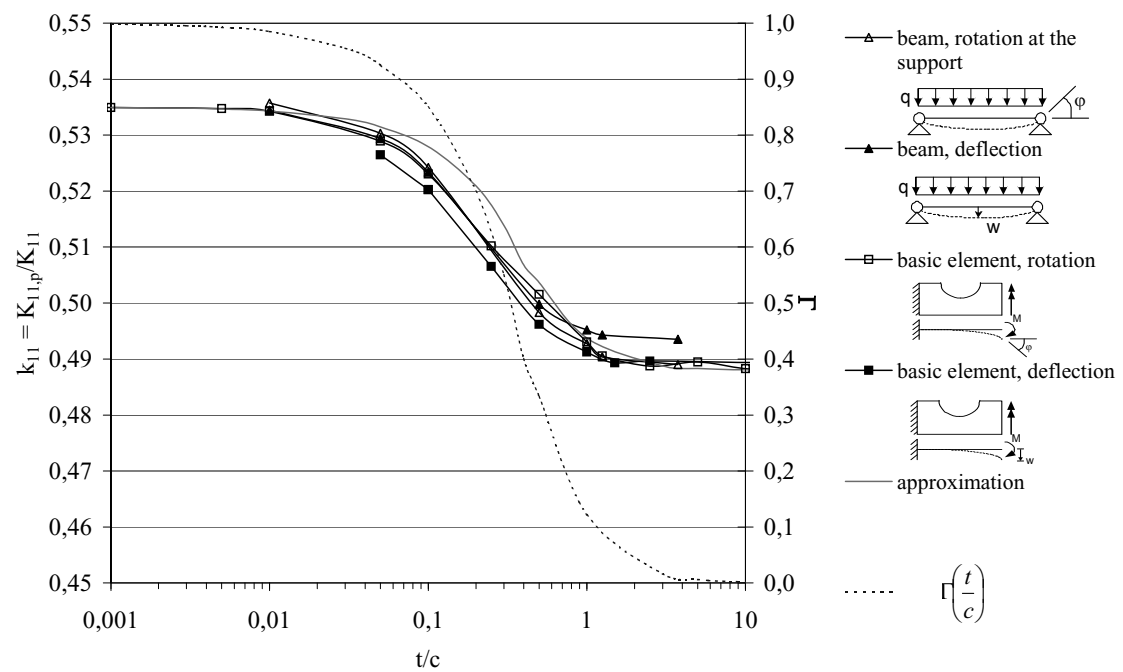

Figure 2: Interpolation between $\mathrm{k}_{11 \text { mint }}$ and $\mathrm{k}_{11 \max t}$ for different values $\mathrm{t} / \mathrm{c}$.

The normalised effective stiffnesses $k_{i j}$ as a function of $t / c$ can be found in [4] - [6] for the different perforation patterns. The effective bending stiffnesses are given for a "very large" and a "very small" plate thickness by $\mathrm{k}_{\max t}$ and $\mathrm{k}_{\min t}$. Then, an interpolation with equations (6) and (7) has to be performed.

\section{LOAD-BEARING CAPACITY OF PLANE CROSS-SECTION PARTS}

The ideal buckling stress of orthotropic rectangular plates under constant uniaxial compressive stress is given by:

$$
\sigma_{c r}=k_{\sigma} \cdot \frac{\pi^{2}}{b^{2}} \cdot \frac{\sqrt{K_{11} \cdot K_{22}}}{t}
$$

as a function of the stiffnesses $\mathrm{K}_{11}$ and $\mathrm{K}_{22}$. The buckling coefficient

$$
k_{\sigma}=\left(\frac{m^{2}}{\bar{\alpha}^{2}}+\frac{\bar{\alpha}^{2}}{m^{2}}\right)+2 \cdot \varsigma
$$


for a rectangular plate with all edges simply supported depends on the corrected aspect ratio

$$
\bar{\alpha}=\alpha \cdot \sqrt[4]{\frac{K_{22}}{K_{11}}}=\frac{a}{b} \cdot \sqrt[4]{\frac{K_{22}}{K_{11}}}
$$

and the stiffness coefficient

$$
\zeta=\frac{K_{12}+2 \cdot K_{44}}{\sqrt{K_{11} \cdot K_{22}}}=\frac{k_{44}+0.3 \cdot\left(k_{12}-k_{44}\right)}{k_{11}}
$$

Using the minimum value of equation (9), the ideal buckling stress of the rectangular plate with all edges simply supported subjected to compression is obtained by

$$
\sigma_{c r, p}=k_{\sigma, p} \cdot \sigma_{e}
$$

with the minimum of the effective buckling value

$$
k_{\sigma, p}=2 \cdot\left[k_{11}+k_{44}+0.3 \cdot\left(k_{12}-k_{44}\right)\right]
$$

This effective buckling coefficient refers to Euler's stress $\sigma_{\mathrm{e}}$ of the unperforated plate. The normalisation with regard to the unperforated plate is only to simplify the application since all reducing influences of the perforation are then included with the buckling coefficient. This simplification is only possible for this case, in which a plate under constant uniaxial compressive stress is treated. The effective buckling coefficient for the actual value of $t / c$ can be plotted versus $d / c$ for the three perforation patterns described in Figure 1. If the values $\mathrm{k}_{\sigma, p, \min t}$ and $\mathrm{k}_{\sigma, \mathrm{p}, \max t}$ are already known for a plate under constant uniaxial compressive stress, the value $\mathrm{k}_{\sigma, \mathrm{p}}$ for the actual value of $\mathrm{t} / \mathrm{c}$ is also obtained by interpolation with the function $\Gamma$ according to (6) and (7) with $\mathrm{k}_{\sigma, \mathrm{p}, \min t}$ and $\mathrm{k}_{\sigma, \mathrm{p}, \max t}$ instead of $\mathrm{k}_{\mathrm{ij}, \min t}$ and $\mathrm{k}_{\mathrm{ij}, \max t}$. The values $\mathrm{k}_{\sigma, p, \min t}$ and $\mathrm{k}_{\sigma, \mathrm{p}, \max t}$ can be taken from Figure 3 depending on ration $\mathrm{d} / \mathrm{c}$, but they can also be calculated with equation (13), using the parameters $\mathrm{k}_{\mathrm{ij}}$ presented in [4] - [6]. Compressive stresses varying along the width of the plate are dealt with in [6].
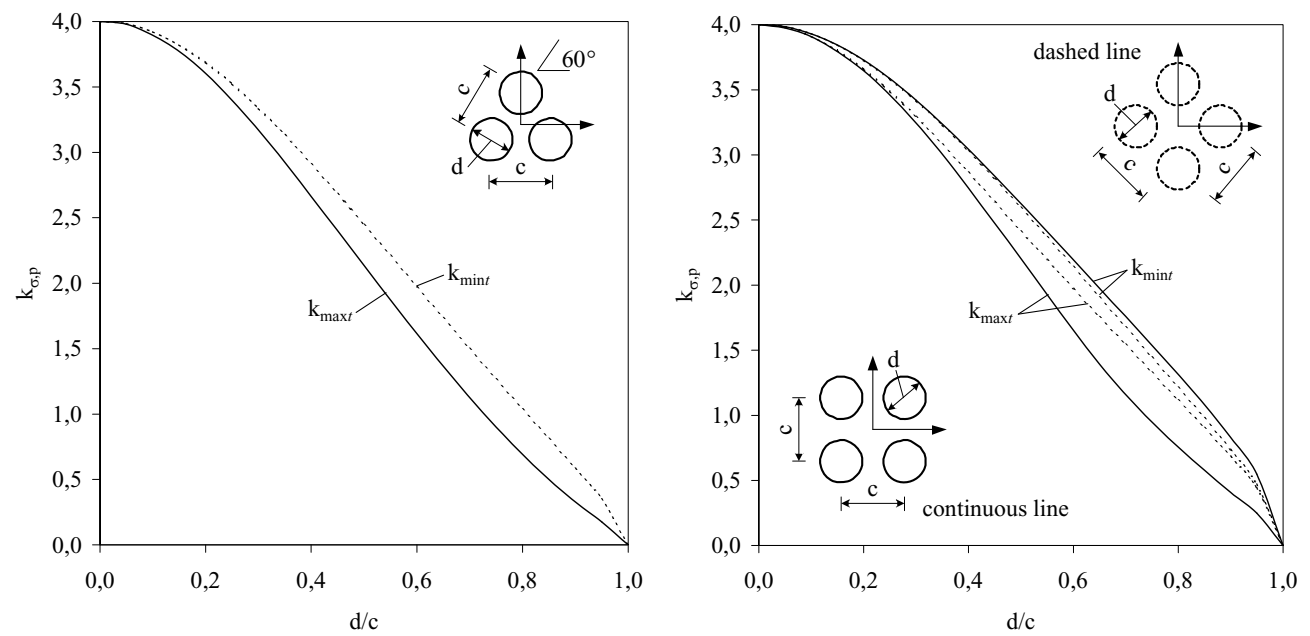

Figure 3: Buckling value $\mathrm{k}_{\sigma, \mathrm{p}}$ for different perforation patterns.

Starting from the effective buckling stress $\sigma_{\mathrm{cr}, \mathrm{p}}$ of the perforated sheet the slenderness for calculating the effective width is determined from

$$
\bar{\lambda}_{p, p}=\sqrt{\frac{c_{0}}{c} \cdot \frac{f_{y}}{\sigma_{c r, p}}}
$$


corresponding to the procedure for the unperforated sheet. The reduction of the yield strength with the factor $\mathrm{c}_{0} / \mathrm{c}$ corresponds to the use of the gross cross-section when determining the stress in the perforated cross-section. When the effective width $b_{\text {eff }}$ is determined, the weakening of the cross-section by the perforation is taken into account with the adaptation of the equation of Winter which for example for steel sheeting is:

$$
\rho=\frac{b_{e f f}}{b}=\frac{c_{0}}{c} \cdot\left(\frac{1}{\bar{\lambda}_{p, p}}-\frac{0.22}{\bar{\lambda}_{p, p}^{2}}\right) \leq \frac{c_{0}}{c}
$$

Equation (15) shows even better than equation (14) that the factor $c_{0} / c$ is necessary to present the controlling net cross-section by the gross cross-section. Since $c_{0} / \mathrm{c}$ can be reduced from (15), there is no change of the limiting slenderness at which no buckling of the perforated sheet occurs and for which yielding becomes controlling:

$$
\bar{\lambda}_{p, \lim } \leq 0.5 \cdot(1+\sqrt{0.12})=0.673
$$

\section{WEB-CRIPPLING}

For trapezoidal profiles, the supporting force at the end support or intermediate support that can be transmitted to the substructure is limited by the failure mode web crippling (Figure 4). This load-bearing capacity primarily depends on the stiffness and strength of the webs. If trapezoidal profiles have a perforation extending completely or partly over the webs, the associated reduction of the cross-section and the stiffness results in a reduced web-crippling resistance.

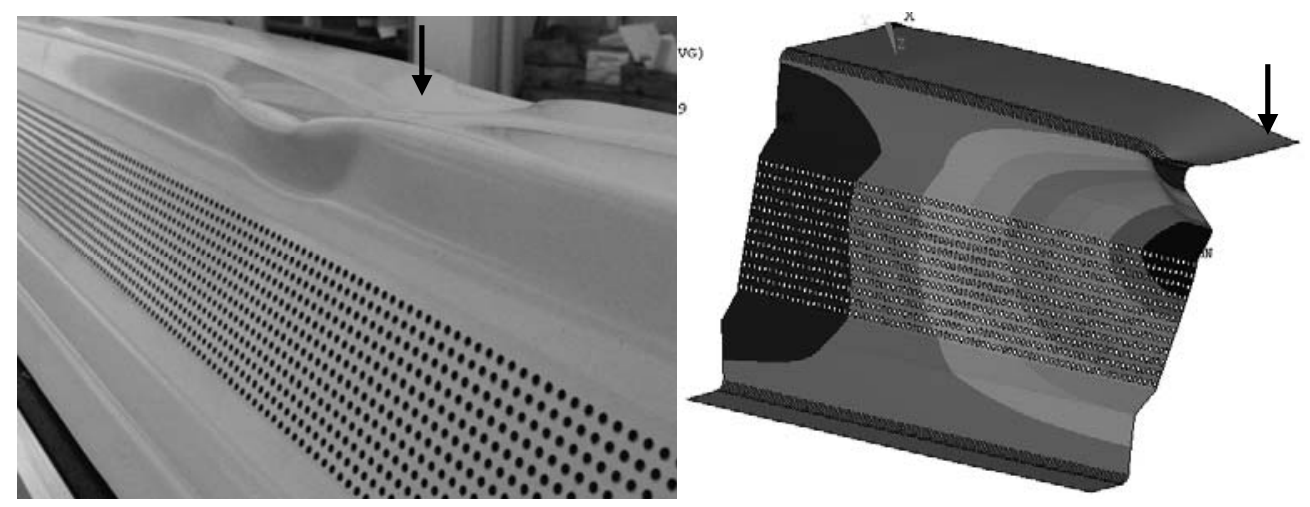

Figure 4: Web-crippling failure at supports.

The equations of EN 1993-1-3 and EN 1999-1-4 for calculating the supporting force for trapezoidal profiles made of steel or made of aluminium respectively can be generalized as follows:

$$
R_{B}=C \cdot t^{2} \cdot C_{f y} \cdot C_{r} \cdot C_{b B} \cdot C_{\phi}
$$

with

C constant factor

$\mathrm{t}$ sheet thickness

$\mathrm{C}_{\mathrm{fy}} \quad$ strength and stiffness

$\mathrm{C}_{\mathrm{r}} \quad$ influence of the bending radii

$\mathrm{C}_{\mathrm{bB}} \quad$ support width or length of the load application

$\mathrm{C}_{\Phi} \quad$ slope of the web relative to the flanges

The factors $C_{i}$ describe - with the exception of the constant factor $C$ as well as the factor $C_{f y}-$ the influence of geometry and load-bearing capacity. The influence of perforation can be included using a 
further factor $\mathrm{C}_{\mathrm{p}}$ for fully perforated trapezoidal profiles. If the failure by web-crippling is considered as a buckling problem with $\mathrm{k}_{\sigma}=4.0$, we obtain with equation (13)

$$
C_{p}=\sqrt{\frac{c_{0}}{c} \cdot \frac{k_{\sigma, p}}{4}}=\sqrt{\frac{c_{0}}{c} \cdot \frac{k_{11}+k_{44}+0.3 \cdot\left(k_{12}-k_{44}\right)}{2}}
$$

Analogous to this factor $\mathrm{C}_{\mathrm{p}}$ for fully perforated webs, a factor $\mathrm{C}_{\mathrm{tp}}$ can be determined for partially perforated webs. The factor $\mathrm{C}_{\mathrm{tp}}$ was determined by FE-analysis, because the influence of the various geometrical parameters demanded for such a detailed study. Figure 5 shows qualitatively how the parameter $\mathrm{C}_{\mathrm{tp}}$ depends on the ratio of width $\mathrm{b}_{1}$ to the support width $\mathrm{b}_{\mathrm{B}}$. The necessary geometrical parameters are also presented in Figure 5.
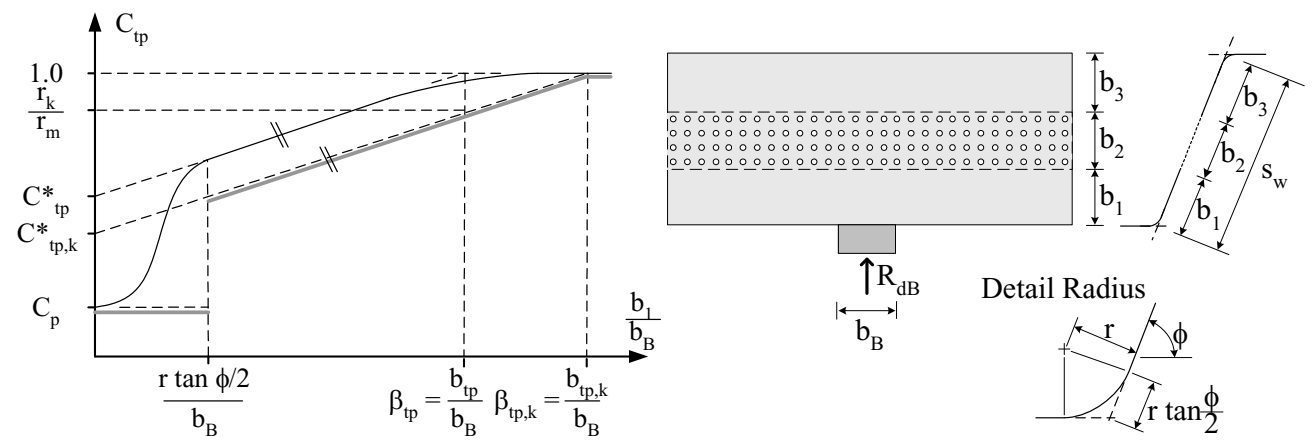

Figure 5: $C_{t p}$ in dependence of $b_{1}$ to $b_{B}$ and the corresponding dimensions of the sheeting.

For large widths $b_{1}$ there is no reduction of the load-bearing capacity. Starting at a width $b_{1}=\beta_{\text {tp }} \cdot b_{B}$ it approximately decreases linearly with decreasing $b_{1} / b_{B}$. This applies until the perforation reaches the radius. From there on with $\mathrm{b}_{1} \leq \mathrm{r} \cdot \tan (\phi / 2)$ a very strong reduction of the load-bearing capacity occurs. For these cases with $b_{1} \leq \mathrm{r} \cdot \tan (\phi / 2)$ the profile can be considered as fully perforated, i.e. the simplifying approach $\mathrm{C}_{\mathrm{tp}}=\mathrm{C}_{\mathrm{p}}$ applies. The results of the numerical investigations presented in [7] lead to:

$$
C_{t p}=\left\{\begin{aligned}
b_{1} & >\beta_{t p} \cdot b \\
\frac{\beta_{1}}{\beta_{t p}} \cdot \frac{s_{w}}{b_{B}}+C_{t p}^{*} \cdot\left(1-\frac{\beta_{1}}{\beta_{t p}} \cdot \frac{s_{w}}{b_{B}}\right) & \text { for } r \cdot \tan \frac{\phi}{2}<b_{1} \leq \beta_{t p} \cdot b_{B} \\
C_{p} & b_{1} \leq r \cdot \tan \frac{\phi}{2}
\end{aligned}\right.
$$

with

$$
C_{t p}^{*}=0.59+0.41 \cdot \sqrt{\frac{c_{0}}{c} \cdot \frac{k_{\sigma, p}}{4}}
$$

and

$$
\beta_{t p}=2.57 \cdot\left(0.70-0.09 \cdot \sqrt{\frac{r}{t}}\right) \cdot\left(0.57-0.37 \cdot\left(\frac{\phi}{90}\right)^{2}\right)
$$

$b_{B}$ has to be limited to $s_{w}$ in equation (19) as well as in the criteria for differentiation. A statistical evaluation was performed to take into account the scatter in results. For design purposes, the characteristic values

$$
C_{t p, k}^{*}=0.92 \cdot C_{t p}^{*}
$$

and 


$$
\beta_{t p, k}=\beta_{t p} \cdot \frac{1-C_{t p, k}^{*}}{1-C_{t p}^{*}}
$$

should be used in connection with equation (19). Thus, the load-bearing capacity results in:

$$
R_{d B, t p}=C_{t p} \cdot R_{d B}
$$

with $\mathrm{R}_{\mathrm{dB}}$ according to EN 1993-1-3 or EN 1999-1-4.

\section{COMPARISON WITH TEST RESULTS}
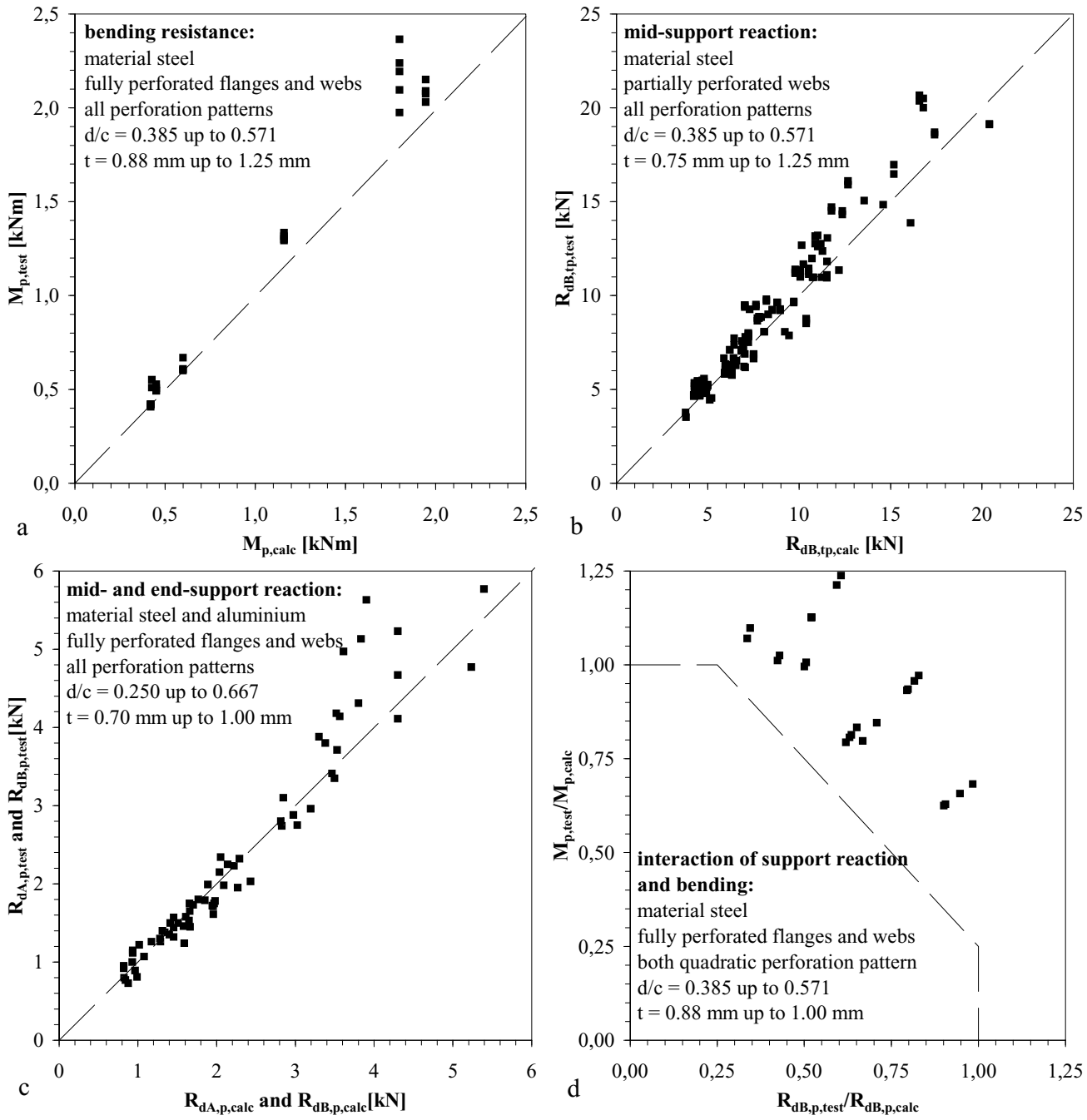

Figure 6: Comparison of calculated load-bearing capacities with test results. 
Figures $6 \mathrm{a}$ to $6 \mathrm{~d}$ show a comparison of calculated load-bearing capacities with test results for different applications. From this it is obvious that theory matches praxis, showing the usual scatter for interaction of support reaction and bending (Figure 6d).

\section{CONCLUSION}

Thin-walled perforated sheeting is used for sound insulation in buildings. Unfortunately, the regulations of the EN 1993-1-3 and EN 1999-1-4 for perforated sheeting are insufficient and partly misleading. This paper overcomes these insufficiencies. The results of these improvements which also take into account the post-buckling resistance is expressed such that the calculation of the load-bearing capacity of thin-walled structural elements being at risk of buckling failure is correct and easy. This is achieved by introducing parameters $\mathrm{k}_{\mathrm{ij}}$ into the design procedures of EN 1999-1-3 and EN 1999-1-4.

Based on comprehensive numerical and experimental investigations on web crippling, it could be shown for fully and partially perforated trapezoidal profiles, that the influence of the perforation can be expressed with the application of a factor $C_{p}$ for fully perforated webs and $C_{t p}$ for partially perforated webs to the capacities of the unperforated sheeting according to EN 1993-1-3 and EN 1999-1-4. A calculation procedure for these factors is described.

In [4], the previously mentioned facts are presented in such a way that they can easily be adopted in design codes. In addition, further topics such as shear buckling and corrugated sheeting are covered.

\section{REFERENCES}

[1] EN 1993-1-3:2006: Eurocode 3: Design of steel structures - Part 1-3: General rules supplementary rules for cold-formed members and sheeting.

[2] EN 1999-1-4:2010: Eurocode 9: Design of aluminium structures - Part 1-4: Cold-formed structural sheeting.

[3] Schardt, R., Bollinger, K., „Zur Berechnung regelmäßig gelochter Scheiben und Platten“ (On the calculation of periodically perforated plates). Bauingenieur, 56(6), 227-239, 1981.

[4] Kathage, K., Misiek, Th., Saal, H., "Stiffness and critical buckling load of perforated sheeting". Thin-Walled Structures, 44(12), 1223-1230, 2006.

[5] Misiek, Th., Saal, H., Kathage, K., "Tragfähigkeit perforierter dünnwandiger Bauteile" (Design resistance of perforated trapezoidal sheeting). Stahlbau, 78(5), 318-323, 2009.

[6] Misiek, Th., Tragverhalten dünnwandiger Bauteile aus perforierten Blechen (Load-bearing behaviour of thin-walled building components made of perforated sheets). Berichte der Versuchsanstalt für Stahl, Holz und Steine der Universität Fridericiana in Karlsruhe, 5. Folge Heft 21, Karlsruhe, Germany, 2008.

[7] Misiek, Th., Albiez, M., "Stegkrüppeln bei voll- und teilperforierten Trapezprofilen" (Web crippling at fully perforated and partly-perforated trapezoidal sections). Stahlbau, 77(2), 87-92, 2008. 